\title{
Solid electrolyte function of a polyethylene porous membrane filled with Side-Chain Crystalline Block co-polymer by using its crystalline supramolecular interaction
}

\author{
Yusuke Sano, Ryoko Nakano, Hiroshi Sekiguchi, Shigeru Yao* \\ Dept. of Chemical Engineering, Fukuoka University, 8-19-1 Nanakuma, Jonan-ku, Fukuoka, Japan, 814-0180
}

Email address:

shyao@fukuoka-u.ac.jp (S. Yao)

To cite this article:

Yusuke Sano, Ryoko Nakano, Hiroshi Sekiguchi, Shigeru Yao. Solid Electrolyte Function of a Polyethylene Porous Membrane Filled with Side-Chain Crystalline Block Co-Polymer by Using Its Crystalline Supramolecular Interaction. International Journal of Materials Science and Applications. Vol. 3, No. 6, 2014, pp. 399-403. doi: 10.11648/j.ijmsa.20140306.28

\begin{abstract}
Side-Chain Crystalline Block Co-Polymer (SCCBC), which is composed of a side-chain crystalline monomer unit and a functional monomer unit (which can confer solvent-compatibility, polarity, etc.), can be adsorbed to a polyethylene crystal through crystalline supramolecular interaction. By using this interaction, we can modify not only the surface but also the inner pores of a porous polyethylene membrane to give various properties. In this study, we used a monomer with ethylene oxide repeating units as a functional unit, and evaluated its potential as a solid electrolyte for use in a Li ion secondary battery. The properties of the solid electrolyte were excellent compared to those of a non-modified porous membrane at low temperature. In addition, the solid electrolyte did not show temperature-dependence, and the $\mathrm{Li}^{+}$ion conductivity remained nearly constant throughout the temperature range of $30^{\circ} \mathrm{C}$ to $60^{\circ} \mathrm{C}$. However, at high temperature (above $70{ }^{\circ} \mathrm{C}$ ), the conductivity began to decrease. This characteristic may make it useful for sensing temperature and for self-controlling thermal runaway.
\end{abstract}

Keywords: Solid Electrolyte, Side-Chain Crystalline Block Co-Polymer, Crystalline Supramolecular Interaction, Filling Membrane

\section{Introduction}

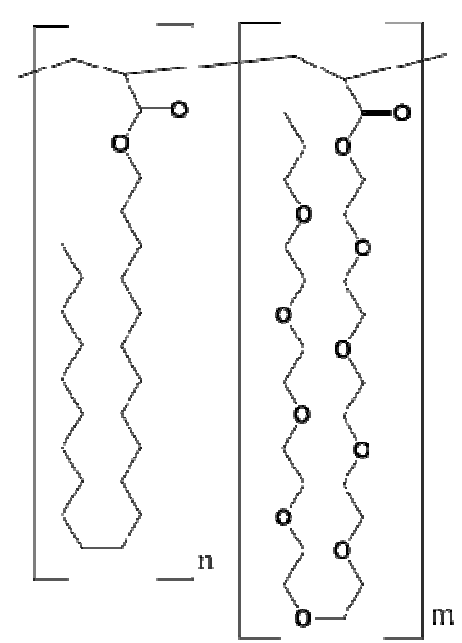

Figure 1. Typical chemical structure of side-chain crystalline block copolymer.
It is well known that a polymer with long alkane side-chains (more than 10 carbon atoms) is a side-chain crystalline polymer [1-3]. Recently, we polymerized a block copolymer composed of a side-chain crystalline monomer unit and a solvent-compatible monomer unit (Side-Chain Crystalline Block Copolymer: SCCBC) by living radical polymerization. Figure 1 shows a typical chemical structure of SCCBC. We found that this SCCBC could be a very good dispersant of concentrated polyethylene (PE) particles dispersion systems [4-9].

This was because the side-chain crystalline unit of this block copolymer is strongly adsorbed on the PE surface, and this adsorption can be considered a form of crystalline supramolecular interaction. Figure 2 shows a schematic illustration of the mechanism of the adsorption of SCCBC on a PE surface. As a result of this adsorption, the surface of the PE particle is covered by solvent-compatible units and is easily wetted by solvent, which eliminates particle aggregation. 


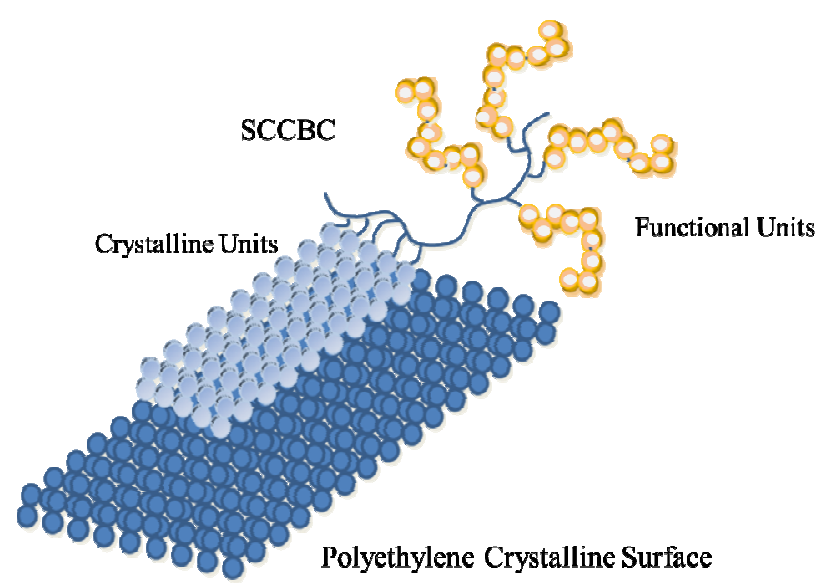

Figure 2. Schematic of the crystalline supramolecular interaction mechanism of the adsorption of SCCBC on a polyethylene surface. The side-chain crystalline units are adsorbed onto the PE crystalline surface and construct a pseudo crystal.

The surface of PE has generally been thought to be inert because of its high crystallinity, and no chemical group that can interact with it has been identified. For the modification of a PE surface, physical modifications (UV or plasma irradiation) have usually been carried out. However, these methods often do not result in the required properties and essentially cleave the polymer chain. Therefore, these methods decrease the mechanical properties of PE and can not be applied to a thin PE film. Furthermore, such methods can not be used to effectively modify the interior of a porous PE membrane.

Even when the solvent-compatible unit of a SCCBC is composed of polar chemical groups, the SCCBC can easily dissolve in an organic solvent because of its side-chain crystalline unit. Thus, a solution that contains the SCCBC can penetrate into the pores of a porous PE membrane, which means that polar chemical units can be placed inside a porous PE membrane. In addition, such polar chemical units can stably remain inside the $\mathrm{PE}$ pores because the side-chain crystalline unit of the SCCBC is strongly adsorbed to the PE surface. In fact, since SCCBC is adsorbed so strongly, all that is necessary for SCCBC to penetrate into the pores is to place the SCCBC on a PE porous membrane and then raise the temperature to above the melting point of SCCBC.

By using this approach, we can modify a PE porous membrane to give functional membranes. In this study, we used a monomer with ethylene oxide repeating units as a functional unit, and evaluated its potential as a solid electrolyte for use in a Li ion secondary battery.

\section{Experimental}

\subsection{Polymerization}

For the side-chain crystalline monomer, we used stearyl acrylate (STA) and behenyl acrylate (BHA). For the functional monomer, we used di(ethylene glycol) ethyl ether acrylate (DEEA) and polyethylene glycol mono acrylate (PGMA). To initiate polymerization, we used 3,7-dioxa-4-aza-6-phosphanonanoic acid, 4,5-bis(1,1-dimethylethyl)-6-ethoxy-2, 2-dimethyl-, 6-oxide (SG-1-MA; BlocBuilder@, ARKEMA). Polymerization was performed under a nitrogen atmosphere at $105^{\circ} \mathrm{C}$. The block copolymer was polymerized by sequential monomer addition. The polymerized SCCBCs and the weight average molecular weight of each unit are summarized in Table 1.

Table 1. Molecular weights of the units of SCCBCs used in this study

\begin{tabular}{lllll}
\hline \multirow{2}{*}{ Sample code } & Crystalline unit & & Functional unit & \\
\cline { 2 - 5 } & Molecular specimen & Molecular weight $(\mathbf{g} / \mathbf{m o l})$ & Molecular specimen & Molecular weight $(\mathbf{g} / \mathbf{m o l})$ \\
\hline SCCBC-1 & STA & 5,000 & DEEA & 9,500 \\
SCCBC-2 & BHA & 3,000 & PGMA & 60,000 \\
\hline
\end{tabular}

\subsection{PE Membrane Modification}

In this study, we used two PE membranes: a PE porous filter (Mykrolis®UPE, pore size $0.1 \mu \mathrm{m}$ ) and a PE separator for a Li ion secondary battery (thickness $30 \mu \mathrm{m}$, porosity $64 \%$ and
Gurle value $78 \mathrm{sec} / 100 \mathrm{cc})$. These membranes were modified by dipping them into dilute $(0.5 \mathrm{wt} \%)$ acetanilide SCCBC solutions. The properties of the solutions are listed in Table 2.

Table 2. Species of Porous PE Membrane, SCCBC, Solution, and Additive for Filling a Porous PE Membrane

\begin{tabular}{lllll}
\hline Sample code & Species of PE porous membrane & SCCBC & Solution & Additive \\
\hline mod-PE1 & PE filter (Mykrolis®UPE) & SCCBC-1 & acetanilide & - \\
mod-PE1 & PE Li ion battery separator & SCCBC-2 & acetanilide & lithium hexafluorophosphate (1mol/l) \\
\hline
\end{tabular}

In the case of the PE separator, we added lithium hexafluorophosphate to the acetanilide solution to give $1 \mathrm{~mol} / \mathrm{l}$. In the case of mod-PE1, we used N,N-dimethyl acetamide as the liquid electrolyte and added lithium hexafluorophosphate to give 1mol/l. After modification, the membranes were air-dried and vacuum-dried before the conductivity measurement.

\subsection{Conductivity Measurement}

$\mathrm{Li}^{+}$ion conductivity was measured by using an LCR-HITESTER impedance device (HIOKI 3522-50). The impedances were measured at 34 frequencies within the range of 50 to $80,000 \mathrm{~Hz}$. For each measurement, we made a Cole-Cole plot and determined the $\mathrm{Li}^{+}$ion conductivity. The 
temperature was varied from $35^{\circ} \mathrm{C}$ to $75^{\circ} \mathrm{C}$.

\section{Results and Discussion}

Figure 3 shows photos of the results of dropping the modified (by dipping) and unmodified PE membranes in water. While the unmodified membrane floated on the water, the modified membrane sank. This indicates that, as a result of simple dipping in SCCBC solution, the PE membrane becomes hydrophilic not only on its surface but also in its interior.

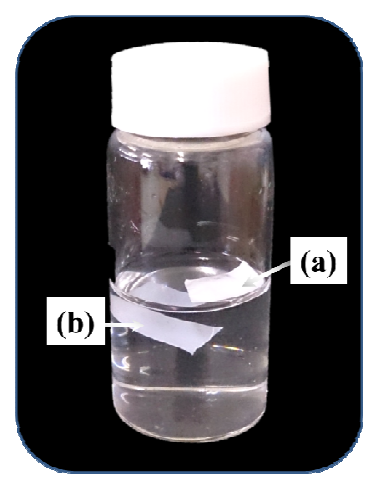

Figure 3. Photo of unmodified (a) and modified (b) PE porous membranes in water.

Figure 4 shows a SEM image of the porous PE membrane after it was filled with the SCCBC by dipping. In the image, the pores are covered with thin films. This suggests that the SCCBC filled the pores and was stabilized due to its affinity with PE. These results show that we can easily modify the $\mathrm{PE}$ membrane to become hydrophilic with the use of the crystalline supramolecular interaction of SCCBC.

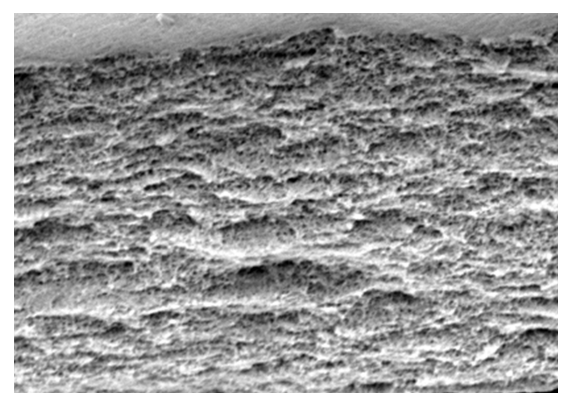

Figure 4. SEM image of the cross-section of a porous PE membrane modified by dipping in dilute SCCBC solution.

Figure 5 shows the temperature-dependence of the $\mathrm{Li}^{+}$ion conductivity of unmodified PE membrane and mod-PE1 membrane using DMAc/LiAlO4 solution as an electrolyte. The $\mathrm{Li}^{+}$ion conductivity of unmodified $\mathrm{PE}$ membrane increased with the temperature. This is a common phenomenon, and is caused by an increase in the mobility of $\mathrm{Li}^{+}$ion and a decrease in the viscosity of the electrolyte. For mod-PE1, the $\mathrm{Li}^{+}$ion conductivity increased at lower temperature. This was because the PE membrane, which was originally hydrophobic and thus showed poor wettability with the electrolytic solution, had become hydrophilic by the adsorption of SCCBC.

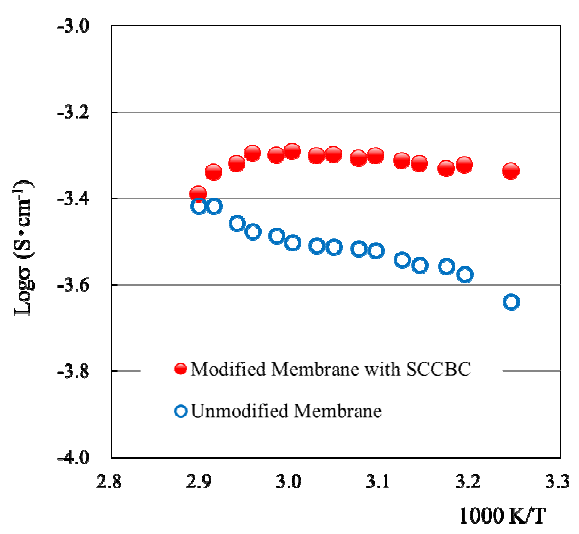

Figure 5. Temperature-dependence of $\mathrm{Li}^{+}$ion conductivity. Open circle shows an unmodified PE membrane used as a separator and closed circle shows a mod-PE1 membrane used as a separator.

Interestingly, for the modified PE membrane, the $\mathrm{Li}^{+}$ion conductivity became almost independent of temperature. Over $65^{\circ} \mathrm{C}$, the conductivity began to decrease, until finally (at about $75^{\circ} \mathrm{C}$ ) the conductivity was almost the same as that of unmodified PE membrane. Figure 6 shows the mechanism of the change in conductivity. At low temperature, the SCCBC is adsorbed on the surface of a pore of the PE membrane, the surface becomes hydrophilic, and $\mathrm{a} \mathrm{Li}^{+}$ion channel is constructed. This is why there is an increase in $\mathrm{Li}^{+}$ion conductivity at low temperature. At high temperature, the SCCBC is desorbed from the surface of PE, the original hydrophilic surface reappears, and the $\mathrm{Li}^{+}$ion channel is closed.

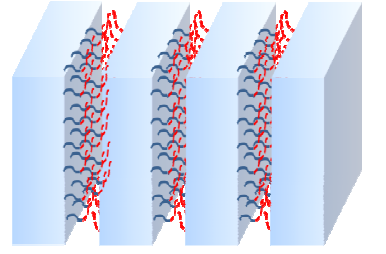

(a)

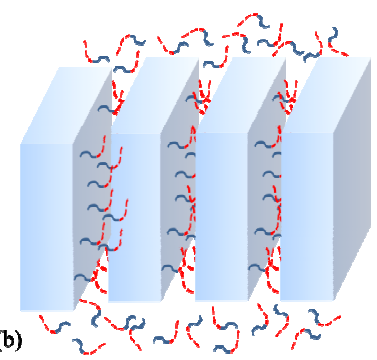

(c)

Figure 6. Schematic images of adsorbed and desorbed SCCBC on the pore surface of a porous PE membrane. (a) low temperature and (b) high temperature. (c) is shcematic modeled SCCBC, solid line represents side chain crystalline units and dashed line represents functional units.

In a previous paper, we estimated the change in diameter by using the Hagen-Poiseuille equation (equation 1).

$$
V=\frac{\pi r^{4} \Delta P}{8 L \eta}
$$

where $\Delta \mathrm{P}:$ decrease in pressure, $\mathrm{L}$ : length of the cylindrical pore, $\eta$ : dynamic viscosity, r: radius, $p$ : mathematical constant, and V: volume of flow per unit time. $\mathrm{V}$ is considered to be proportional to the $\mathrm{Li}^{+}$ion conductivity. Therefore, the 
difference in conductivity is associated with the pore diameter of the $\mathrm{Li}^{+}$ion channel (equation 2).

$$
\frac{S_{1}}{S_{0}}=\frac{V_{1}}{V_{0}}=\left(\frac{d_{1}}{d_{0}}\right)^{4}
$$

where $\mathrm{d} 1$ and $\mathrm{d} 2$ are the effective pore diameters of the $\mathrm{Li}^{+}$ion channel.

If we apply this equation in this case, we see that, upon modification with SCCBC at low temperature, the diameter increased 1.2-fold, and the diameter decreased 0.96-fold with the desorption of SCCBC. We might be able to use this phenomenon to determine the temperature of a $\mathrm{Li}$ ion battery.

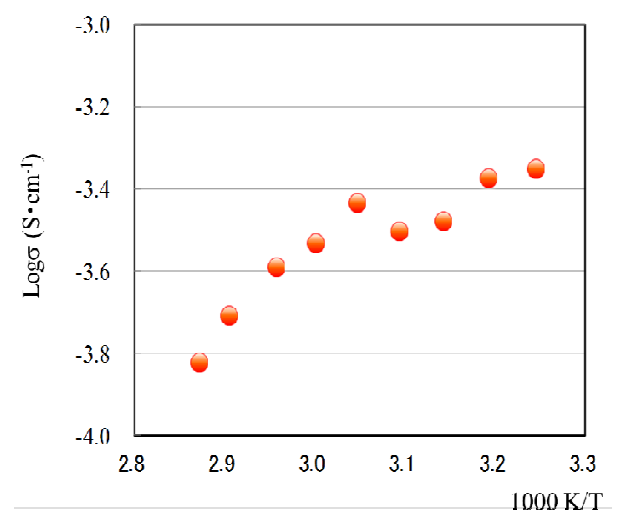

Figure 7. Temperature-dependence of $\mathrm{Li}^{+}$ion conductivity of a mod-PE2 membrane used as a solid electrolyte.

Furthermore, a battery might be able to automatically decrease its output capacity at high temperature.

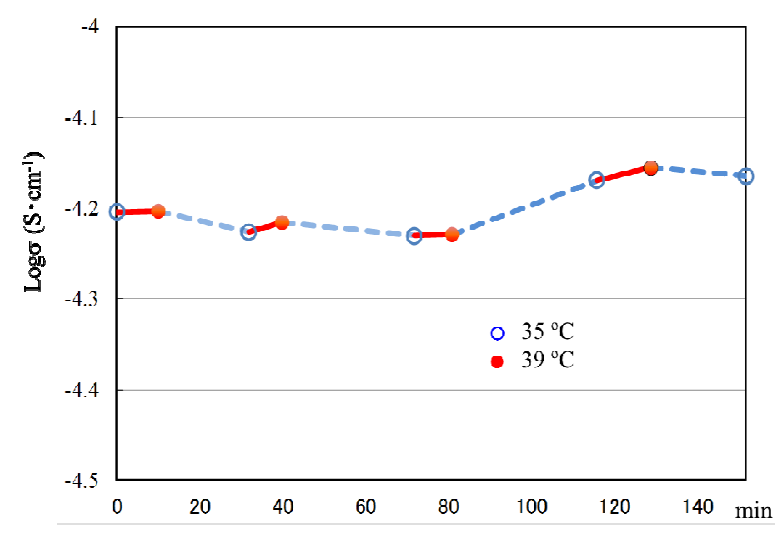

Figure 8. Time- and temperature-dependence of $\mathrm{Li}^{+}$ion conductivity of a mod-PE2 membrane used as a solid electrolyte. The temperature was cycled between $35^{\circ} \mathrm{C}$ and $39^{\circ} \mathrm{C}$. Solid lines mean heating and dashed lines mean cooling.

Figure 7 shows the solid electrolyte ability of mo-PE2 modified by SCCBC-2. The conductivity at $35{ }^{\circ} \mathrm{C}$ was $4.47 \times 10^{-4} \mathrm{~S} \bullet \mathrm{cm}$, which is very high. In this case, due to the side-chain crystalline unit adsorbed on the side wall of the PE pore surface, the polyoxy ethylene layer was constructed parallel to the pore side wall from the upper surface to the lower surface of the membrane.

In this case, the temperature-dependence is slightly negative at the lower and middle temperature range and decreases rapidly at high temperature. The same mechanism that is at work in Fig. 5 may be having an effect here.

Figure 8 shows the stability of conductivity at a lower temperature range. We evaluated the conductivity under heating and cooling cycles between $35^{\circ} \mathrm{C}$ to $39^{\circ} \mathrm{C}$. Within this temperature range, the conductivity is very stable. This result suggests that a PE membrane filled with SCCBC would be a very excellent solid electrolyte under normal conditions, and could decrease the conductivity at high temperature.

\section{Conclusions}

The SCCBC is strongly adsorbed onto PE crystal by its crystalline supramolecular interaction. It also readily dissolves in organic solvent. By using these properties, we can modify the hydrophobic property of the surface and the pores of a PE membrane simply by dipping the membrane into a SCCBC solution. The original hydrophobicity of the PE membrane can be restored at high temperature.

In this study, we modified a PE membrane so that it would exhibit $\mathrm{Li}^{+}$ion conductivity by using two SCCBCs with ethylene oxide structures. These modified membranes showed excellent separator and solid electrolyte properties. In addition, these membranes had specific properties which suggest that they could be used to detect a temperature change and to automatically decrease the conductivity at high temperature. These properties would be very useful for avoiding the thermal runaway of a Li ion secondary battery.

By changing the functional unit of the SCCBC and applying the associated properties, we may be able to create many other functional filters or separator membranes.

\section{References}

[1] K. Shiratsuchi, T. Matsumoto, S. Yao, N. Shiraishi, "Dynamic viscoelastic properties in the molten state for cellulose acylates and (cellulose oligo-oxymethylene ether) acrylates," J. Appl. Polym. Sci., vol. 34, 1987, pp. 1217-1234.

[2] K. Inomata, Y. Sakamaki, T. Nose, S. Sasaki, "Solid-State Structure of Comb-Like Polymers Having n-Octadecyl Side Chains I. Cocrystallization of Side Chain with n-Octadecanoic Acid,” Polym. J., vol. 28, 1996, pp. 986-991.

[3] Y. Inoue, M. Matsui, S. Kuroki, I. Ando, "A study of diffusional behavior of polymer having semiflexible main-chain with long n-alkyl side-chains as associated with structural behavior of the side-chains,” J. Mol. Struct., vol. 976, 2010, pp. 141-149.

[4] T. Okuma, S. Yao, R. Nakano, H. Sekiguchi, S. Ichikawa, D. Tatsumi, "Supramolecular function of side chain crystalline block co-polymer -Dispersant effect of concentrated polyethylene particle dispersion and thermal rheological effect-," IEICE Technical Report, vol. 113/167, 2013, pp. 81-82.

[5] S. Yao, T. Okuma, C. Kumamaru, H. Sekiguchi, S. Ichikawa, D. Tatsumi, "Supramolecular Interaction of Side-Chain Crystalline Block Co-Polymer and Its Thermal Rheological Function," Mater. Trans. vol. 54, 2013, pp. 1381-1384. 
[6] S. Yao, M. Sakurai, H. Sekiguchi, H. Otsubo, T. Uto, Y. Yamachika, W. Ishino, S. Ichikawa, D. Tatsumi, "Thermal Rheological Fluid Properties of Particle Dispersion Systems using Side Chain Crystalline Block Copolymer (III)," Nihon Reoroji Gakkaishi (J. Soc. Rheol. Japan), vol. 41, 2013, pp. $7-12$.

[7] S. Yao, M. Sakurai, H. Sekiguchi, H. Otsubo, T. Uto, Y. Yamachika, W. Ishino, S. Ichikawa, D. Tatsumi, "The Intelligent Material Function of Side Chain Crystalline Block Copolymer (IV). Control the Lithium Ion Mobility in
Polyethylene Porous Membrane," Nihon Reoroji Gakkaishi (J. Soc. Rheol. Japan), vol. 40, 2012, pp. 253-256.

[8] S. Ichikawa, S. Yao, "Thermal Rheological Properties of the Particle Dispersion Sysytems with Using Side Chain Crystalline Block Co-Polymer II," Nihon Reoroji Gakkaishi (J. Soc. Rheol. Japan), vol. 40, 2012, pp. 37-40.

[9] S. Yao, S. Ichikawa, "A Nobel Dispersant for High Content Polyethylene Particle Dispersion," Nihon Reoroji Gakkaishi (J. Soc. Rheol. Japan), vol. 39, 2011, pp. 181-182 